\title{
THE BOOLEAN RING GENERATED BY A DISTRIBUTIVE LATTICE
}

\author{
S. J. BERNAU ${ }^{1}$
}

\begin{abstract}
This note gives a simple characterization of the Boolean ring $R$ generated by a distributive lattice $L$. The method is to introduce a Hausdorff topology on the Stone representation space $E$ of $L$ and note that $R$ is the set of subsets of $E$ which are compact and open with respect to this topology.
\end{abstract}

Let $L$ be a distributive lattice. Since the adjunction of a least element to $L$, if there is not one already, is an order-theoretic triviality we assume $L$ has a least element.

An ideal of $L$ is a nonempty proper subset $I$ of $L$ such that $x \vee y \in I$ if $x \in I$ and $y \in I$, and $y \in I$ if $x \in I$ and $y \leqq x$. An ideal $I$ of $L$ is prime if $x \wedge y \notin I$ whenever $x \notin I$ and $y \notin I$. The Stone space, $E$, of $L$ is the set of prime ideals of $L$ equipped with the hull-kernel topology. A base for the topology of $L$ is the set of all $U_{a}=\{\theta \in E: a \notin \theta\}(a \in L)$. The map $a \mapsto U_{a}$ is a lattice isomorphism of $L$ onto the set of all compact open subsets of $E$.

Once we have $L$ represented as a lattice of sets it is clear that $L$ can be embedded in a Boolean ring. Indeed the representation by sets and subsequent embedding in a Boolean ring go back to Hausdorff [3], Birkhoff [2], Stone [6] and MacNeille [5]. Our variant of the process stems from recent work on structure spaces of lattice groups [1], a little of which has direct application here.

Consider the dual ordering of $L$. The set of prime dual ideals is exactly the set of complements of prime ideals of $L$. Thus $E$ carries a second natural topology, the dual hull-kernel topology. A base for this is easily seen to be the set of all $V_{a}=\{\theta \in E: a \in \theta\}=E \sim U_{a}(a \in L)$.

Let $E_{S}$ denote $E$ with the supremum of the hull-kernel and dual hullkernel topologies. It is trivial to check that $E_{S}$ is a Hausdorff space. Our main result is:

THEOREM 1. In $E_{S}, U_{a}$ is compact $(a \in L)$.

Received by the editors May 27, 1971.

AMS 1969 subject classifications. Primary 0650; Secondary 0660, 1630.

Key words and phrases. Distributive lattice, Stone representation space, dual hullkernel topology, embedding in a Boolean ring.

${ }^{1}$ This research was partially supported by the National Science Foundation Grant GP-27916.

(c) American Mathematical Society 1972 
Proof. By Alexander's subbase theorem [4] it is sufficient to show that if $\mathscr{C}=\left\{U_{x}: x \in X\right\} \cup\left\{V_{y}: y \in Y\right\}$ is a cover of $U_{a}$ then a finite subset of $\mathscr{C}$ covers $U_{a}$. Suppose this is false.

We claim that the ideal $I$ generated by $X$ and the dual ideal $J$ generated by $Y \cup\{a\}$ do not intersect. If they do there exist $x_{1}, \cdots, x_{n} \in X$ and $y_{1}, \cdots, y_{n} \in Y$ such that $x_{1} \vee \cdots \vee x_{n} \geqq a \wedge y_{1} \wedge \cdots \wedge y_{m}$, then

$$
U_{x_{1}} \cup \cdots \cup U_{x_{n}}=U_{x_{1} \vee \cdots \vee x_{n}} \supset U_{a \wedge y_{1} \wedge \cdots \wedge y_{m}}=U_{a} \cap\left[U_{y_{1}} \cap \cdots \cap U_{y_{m}}\right] \text {; }
$$

and hence $U_{a} \subset U_{x_{1}} \cup \cdots \cup U_{x_{n}} \cup V_{y_{1}} \cup \cdots \cup V_{y_{m}}$ contrary to hypothesis.

It follows that there is an ideal $\theta$ of $L$ maximal with respect to $X \subset \theta$, $\theta \cap J=\varnothing$. Then if $d \notin \theta, b \notin \theta$, there exist $p, q \in \theta$ and $u, v \in J$ such that $d \vee p \geqq u$ and $b \vee q \geqq v$; then $(d \wedge b) \vee p \vee q \geqq u \wedge v$ and $d \wedge b \notin \theta$. Hence $\theta$ is prime, $\theta \in U_{a}$ and $\theta$ is in no set of $\mathscr{C}$. Thus $\mathscr{C}$ is not a cover of $U_{a}$.

Let $R$ denote the set of compact open subsets of $E_{S}$. If $M \in R$ then $M$ is a union, and hence a finite union, of sets $U_{a} \cap V_{b}$ with $a, b \in L$. Thus $R$ is the Boolean subring of $\mathscr{P}\left(E_{S}\right)$ generated by the set $\left\{U_{a}: a \in L\right\}$.

We can easily prove, using the techniques of [5, pp. 454, 455], the following result.

THEOREM 2. If $L$ is identified with its image in $R$ then any lattice homomorphism of $L$ into a Boolean ring $K$ can be uniquely lifted to a Boolean ring homomorphism of $R$ into $K$.

This completes our description of the Boolean ring generated by $L$.

ADDED IN PROOF. These results were obtained independently but are essentially contained in a paper by H. A. Priestley, Representation of distributive lattices by ordered Stone spaces, Bull. London Math. Soc. 2 (1970), 186-190. His approach and ours are different.

\section{REFERENCES}

1. S. J. Bernau, Topologies on structure spaces of lattice groups, Pacific J. Math. (to appear).

2. G. Birkhoff, Lattice theory, 3rd ed., Amer. Math. Soc. Colloq. Publ., vol. 25, Amer. Math. Soc., Providence, R.I., 1967. MR 37 \#2638.

3. F. Hausdorff, Mengenlehre, 2nd ed., de Gruyter, Berlin, 1927.

4. J. L. Kelley, General topology, Van Nostrand, Princeton, N.J., 1955. MR 16, 1136.

5. H. M. MacNeille, Extension of a distributive lattice to a Boolean ring, Bull. Amer. Math. Soc. 45 (1939), 452-455.

6. M. H. Stone, Topological representations of distributive lattices and Brouwerian logics, Casopis Pěst. Mat. Fys. 67 (1937), 1-25.

Department of Mathematics, University of Texas, Austin, Texas 78712 